\title{
Candida glabrata and Candida albicans co-infection of an in vitro oral epithelium
}

\author{
Sónia Silva ${ }^{1,3}$, Mariana Henriques ${ }^{1}$, Anthony Hayes ${ }^{2}$, Rosário Oliveira ${ }^{1}$, Joana Azeredo ${ }^{1}$, \\ David W. Williams ${ }^{3}$ \\ ${ }^{1}$ Institute for Biotechnology and Bioengineering, Universidade do Minho, Campus de Gualtar 4710-057, Braga, Portugal; \\ ${ }^{2}$ School of Biosciences, Cardiff University, Park Place, Cardiff, CF10 3US, UK, ${ }^{3}$ Tissue Engineering and Reparative Dentistry, \\ School of Dentistry, Cardiff University, Heath Park, Cardiff, CF14 4XY,UK
}

\begin{abstract}
BACKGROUND: Candida albicans is regarded as the leading of candidosis. However, Candida glabrata has emerged as an important pathogen of oral mucosa, occurring both singly or in mixed species infections, often with C. albicans. Compared with C. albicans, little is known about the role of $C$. glabrata in oral infection. The aim of this study was to examine single and mixed species infection of oral epithelium involving C. glabrata and establish its ability to invade and damage tissue.
\end{abstract}

METHODS: A reconstituted human oral epithelium (RHOE) was infected only with $C$. glabrata, or simultaneously with $C$. glabrata and $C$. albicans. The ability of both species to invade the tissue was examined using species specific peptide nucleic acid (PNA) probe hybridization and confocal laser scanning microscopy. Epithelial damage was assessed by measuring lactate dehydrogenase (LDH) activity.

RESULTS: Candida glabrata strains were able to colonize the RHOE, in a strain dependent manner. Candida glabrata single infection after $12 \mathrm{~h}$, generally revealed no invasion of the RHOE, which contrasted with extensive tissue invasion demonstrated by $C$. albicans. Mixed infection showed that $C$. albicans enhanced the invasiveness of C. glabrata, and led to increased LDH release by the RHOE, which paralleled the observed histological damage.

CONCLUSIONS: The results obtained demonstrating enhanced invasion and increased tissue damage caused by mixed C. glabrata and C. albicans infections have important clinical significance and highlight the need to identify Candida species involved in oral candidosis.

J Oral Pathol Med (20II) 40: 42I-427

Keywords: candidosis; co-infection; Candida albicans; Candida glabrata; yeast PNA FISH

Correspondence: Mariana Contente Henriques, Institute for Biotechnology and Bioengineering, Universidade do Minho, Campus de Gualtar 4710-057, Braga, Portugal. Tel: + 351 253604408, Fax: + 351 253678986, E-mail:mcrh@deb.uminho.pt

Accepted for publication November 3, 2010

\section{Introduction}

Candidosis is a frequent opportunistic infection in immunocompromised individuals (1). Candida albicans remains the main aetiological agent in oral candidosis, accounting for $70-80 \%$ of isolates from oral mucosal lesions (2,3). However, in recent years, Candida glabrata has emerged as a notable pathogenic agent of the oral mucosa, either co-infecting with C. albicans (4-7), or occurring in single species infections $(2,4-6)$. Furthermore, $C$. glabrata is the third most common cause of mortality in hospitals (8). Candida glabrata exhibits significant differences from $C$. albicans, including relative physical size, morphological properties and biochemistry. Candida glabrata yeasts are smaller $(1-4 \mu \mathrm{m})$ than $C$. albicans yeast $(4-6 \mu \mathrm{m})$ and have a much narrower spectrum of carbohydrate utilization (8). Furthermore, in contrast to $C$. albicans, $C$. glabrata is generally not polymorphic, and only occurs in the yeast form within the oral cavity (8). One possible explanation therefore for the relatively frequent occurrence of co-infection of C. albicans and C. glabrata is that these significant differences limit the extent of inter-species competition allowing the organisms to occupy similar oral niches. Importantly, C. glabrata-associated oropharyngeal candidosis in HIV and cancer patients tends to be more severe and more difficult to treat than infections caused solely by $C$. albicans $(5,6,9)$.

An understanding of the interactions between these Candida species has significance for the development of strategies for oral candidosis management. This is important since, although oral Candida infections are not associated with mortality, they are a major cause of morbidity, and trigger chronic pain or discomfort upon mastication, which may limit nutritional intake in immunocompromised or elderly individuals $(5,10)$. In addition, oral carriage of Candida has also been proposed to be a pre-disposing factor for systemic infection in severely immunocompromised patients (11).

Fluorescent in situ hybridization (FISH) is rapidly becoming established as a valuable research and 
diagnostic tool in molecular pathology laboratories, having previously been used to detect and differentiate microorganisms in histological sections (12-14). Peptide nucleic acid (PNA) probes are synthetic equivalents of nucleic acids where the phosphate-sugar polynucleotide backbone has been substituted with a peptide polymer which provides the basis for linking nucleobases. Unlike DNA, the peptide backbone of PNA probes is not charged and this offers greater stability in nucleic acidprobe hybridization. Furthermore, PNA probes are more resistant to degradation from nuclease and protease activity. Given such advantages, it is not surprising that PNA probes have emerged as valuable tools in the study of infectious diseases and represent a new generation of therapy-directed diagnostics (14-16). To exemplify this, the Yeast Traffic Light PNA FISH ${ }^{\mathrm{TM}}$ (YTL; AdvanDx, Inc., Woburn, MA, USA) system is a newly developed and commercialized PNA probe kit for the rapid detection and distinction of the five most common and clinically relevant Candida species $(17,18)$.

Previously, a reconstituted human oral epithelium (RHOE) was successfully used to investigate colonization and infection of tissue by Candida (19-23). Coupled with species specific PNA probes and confocal laser scanning microscopy (CLSM), co-infection of RHOE using C. glabrata and $C$. albicans can therefore be readily examined. The aim of this study was to generate single and mixed species infections of RHOE with C. albicans and C. glabrata and ascertain the role of C. glabrata in the infection using species specific PNA probes, CLSM and lactose dehydrogenase (LDH) activity (as a measure of tissue damage) in infected tissue.

\section{Materials and methods}

Organisms

A total of six clinical isolates of C. glabrata, originally recovered from the oral cavity (strains D1 and AE2), vagina (strains 534784 and 585626) and urinary tract (strains 562123 and 513100) were used in this study. A reference strain of C. glabrata from the American Type Culture Collection (ATCC 2001) was also included. Oral isolates were obtained from the biofilm group of the Centre of Biological Engineering, Minho University (Braga, Portugal). Strains isolated from urinary and vaginal infections were kindly provided from the culture collection of the Hospital of São Marcos (Braga, Portugal). An oral isolate of C. albicans (strain 324LA/94) from the culture collection of Cardiff Dental School (Cardiff, UK) was selected based on its ability to invade RHOE (21). The identity of all isolates was initially confirmed by PCR-based sequencing using specific primers (ITS1 and ITS4) for the 5.8S subunit gene (24).

\section{Growth conditions}

For each experiment, isolates were cultured on Sabouraud Dextrose Agar (SDA) (Merck, Darmstadt, Germany) for $48 \mathrm{~h}$ at $37^{\circ} \mathrm{C}$. Isolates were then subcultured in Yeast Nitrogen Base medium (YNB; BD
Diagnostics, Cowley, UK) supplemented with $0.5 \%$ glucose $(\mathrm{w} / \mathrm{v})$ for $12 \mathrm{~h}$ at $37^{\circ} \mathrm{C}$. After incubation, cells were harvested by centrifugation and washed three times with phosphate buffered saline (PBS; pH 7). The yeasts were then enumerated using an Improved Neubauer haemocytometer and adjusted to a concentration of $2 \times 10^{6} \mathrm{cells} / \mathrm{ml}$ in MCDB-153 defined medium (Clonetics, San Diego, CA, USA).

\section{Reconstituted human oral epithelium infection}

To investigate in vitro single- and mixed species infection of oral mucosa by $C$. glabrata and C. albicans strains, a reconstituted human oral epithelium (RHOE) (human keratinocytes derived from a squamous carcinoma of the buccal mucosa, cell line TR 146, SkinEthic Laboratories, Nice, France) model was used. RHOE tissue inserts $\left(0.5 \mathrm{~cm}^{2}\right)$ were placed in 24-well tissue culture plates with MCDB 153-defined medium containing $5 \mu \mathrm{g} / \mathrm{ml}$ insulin, $1.5 \mathrm{mM} \mathrm{CaCl} 2,25 \mu \mathrm{l} / \mathrm{ml}$ gentamicin, and $0.4 \mu \mathrm{g} / \mathrm{ml}$ hydrocortisone, according to the manufacturer's instructions. For single species infection, $1 \mathrm{ml}$ of standardized suspensions $\left(2 \times 10^{6}\right.$ cells $\left./ \mathrm{ml}\right)$ of $C$. glabrata or $C$. albicans was placed directly onto the RHOE tissue inserts. For mixed species infection, $500 \mu \mathrm{l}$ of C. glabrata $\left(2 \times 10^{6}\right.$ cells $\left./ \mathrm{ml}\right)$ was combined with $500 \mu \mathrm{l}$ of $C$. albicans $\left(2 \times 10^{6}\right.$ cells $\left./ \mathrm{ml}\right)$ suspension and this preparation placed onto the RHOE tissue inserts. Control samples were inoculated with $1 \mathrm{ml}$ of medium devoid of Candida. Infected tissues were incubated at $37^{\circ} \mathrm{C}$ in a $5 \% \mathrm{CO}_{2}$ environment with saturated humidity for $12 \mathrm{~h}$. After $12 \mathrm{~h}$ incubation, the maintenance medium was removed and the RHOE tissues rinsed twice with PBS to remove non-adherent Candida cells. The RHOE specimens were then fixed in $2 \%(\mathrm{v} / \mathrm{v}$, in ultrapure water) paraformaldehyde for $24 \mathrm{~h}$, at $4{ }^{\circ} \mathrm{C}$ and embedded in paraffin wax using standard histological techniques. Tissue sections $(20 \mu \mathrm{m})$ were cut and placed onto Histobond + coated microscope slides (Raymond A Lamb, East Sussex, UK), de-waxed and processed through xylene, ethanol and water.

\section{Peptide nucleic acid fluorescent in situ hybridization ( PNA FISH)}

The Yeast Traffic Light PNA FISH ${ }^{\mathrm{TM}}$ (YTL-PNA FISH) kit was used to examine invasion of the RHOE by $C$. glabrata and $C$. albicans. The Candida PNA FISH assay was performed according to the manufacturer's instructions with some modifications $(17,18)$. Sections of RHOE were prepared as described above and then washed briefly in pre-warmed $1 \times$ wash solution. Hybridization with PNA probes was performed using 1 drop of $C$. albicans/C. glabrata PNA probe mixture and the overlay incubated in the dark in a humidified chamber for $90 \mathrm{~min}$ at $55^{\circ} \mathrm{C}$. After probe hybridization, excess PNA probe was removed by immersion of the slides into pre-warmed $1 \times$ wash solution $\left(55^{\circ} \mathrm{C}\right)$ for $30 \mathrm{~min}$. For nuclear staining, keratinocytes within the RHOE sections were counterstained with Hoechst 33258 for $30 \mathrm{~min}$ at room temperature, before washing in $1 \times$ wash solution. The preparation was then mounted using 
Vectashield $^{\circledR}$ fade-retarding mountant (Vector Laboratories, Peterborough, UK).

\section{Confocal laser scanner microscopy}

Sections hybridized with PNA probes were observed by CLSM using a Leica TCS SP2 AOBS spectral confocal microscope (Leica, Wetzlar, Germany). The sections were scanned through the full depth using appropriate settings for single, double or triple channel fluorescence recordings. Fluorescein-labelled PNA was used for detection of $C$. albicans (laser excitation line $492 \mathrm{~nm}$ and emissions detected $520 \mathrm{~nm}$ ), Tamara-labelled PNA was used for detection of $C$. glabrata (laser excitation line $565 \mathrm{~nm}$ and emissions detected $580 \mathrm{~nm}$ ) and Hoechst 33258 (laser excitation line $485 \mathrm{~nm}$ and emissions detected 410-485 nm) for nuclear context of keratinocytes. For multi-channel recordings, fluorochromes were scanned sequentially to eliminate spectral overlap between probes. Selected images were presented either as single confocal optical sections or maximum intensity type constructions. Candida glabrata and C. albicans were identified by red and green colouration respectively, whilst keratinocytes were depicted blue. All experiments were performed in duplicate.

\section{Lactate dehydrogenase activity}

The release of LDH from RHOE into the culture medium was used as a measure of epithelial cell damage. The LDH concentration in the medium from control and infected tissues was determined after $12 \mathrm{~h}$ using the CytoTox-ONE ${ }^{\mathrm{TM}}$ kit (Promega, Madison, WI, USA) according to the manufacturer's instructions. The LDH activity of Candida cells in cell culture medium devoid of tissue was subtracted from the LDH activity of the tissue infected with yeasts. The LDH released during single or co-infection with the different $C$. glabrata strains and C. albicans was then expressed in relation to the untreated control tissue. All experiments were performed in duplicate.

\section{Statistical analysis}

Results were compared using one-way analysis of variance (ANOVA) by applying Levene's test of homogeneity of variance and the Tukey multiple-comparisons test, using SPSS software [SPSS 17 (Statistical Package for the Social Sciences), Inc., Chicago, IL, USA]. All tests were performed with a confidence level of $95 \%$.

\section{Results}

Candida glabrata and Candida albicans single species RHOE infection

The ability of $C$. glabrata and $C$. albicans strains to colonize and invade human oral epithelium was examined after $12 \mathrm{~h}$ of infection using the RHOE model. Results showed that all strains colonized the RHOE surface, although the extent of colonization was highly strain dependent (Table 1 and Fig. 1). Candida glabrata 534784 and 585626 (both vaginal strains) and $C$. albicans exhibited the highest level of RHOE colonization, whilst C. glabrata D1 and AE2 (both oral strains)
Table 1 Candida glabrata and $C$. albicans single and co-infection profile of reconstituted human oral epithelium (at $12 \mathrm{~h}$ ), as assessed using confocal laser scanning microscopy and peptide nucleic acid probe hybridization

\begin{tabular}{|c|c|c|c|c|}
\hline \multirow[b]{2}{*}{ Candida } & \multirow[b]{2}{*}{ Origin } & \multicolumn{2}{|c|}{ Single infection } & \multirow{2}{*}{$\frac{\text { Co-infection }}{\text { Invasion }}$} \\
\hline & & Colonization & Invasion & \\
\hline C. glabrata $(\mathrm{D} 1)$ & Oral & + & None & Moderate \\
\hline C. glabrata (AE2) & & + & None & Moderate \\
\hline C. glabrata (562123) & Urinary & + & None & Moderate \\
\hline C. glabrata $(513100)$ & & ++ & Moderate & High \\
\hline C. glabrata (534784) & Vaginal & +++ & None & Moderate \\
\hline C. glabrata (585626) & & +++ & None & High \\
\hline $\begin{array}{l}\text { C. glabrata } \\
\text { (ATCC 2001) }\end{array}$ & Reference & ++ & None & High \\
\hline $\begin{array}{l}\text { C. albicans } \\
\text { (324LA/94) }\end{array}$ & Oral & +++ & High & - \\
\hline
\end{tabular}

and 562123 (urinary isolate) colonized the tissue to a lower extent (Fig. 1).

In general, after $12 \mathrm{~h}$ of infection, only clusters of C. glabrata yeast were detected at the surface of the keratinocyte layers (Fig. 1A,B). Furthermore, strains of C. glabrata were generally unable to invade the epithelium, although C. glabrata 513100 (urinary isolate), was found within the upper (second or third epithelial cell layer) region of the RHOE (Fig. 1B). In contrast, $C$. albicans showed a greater ability to invade the RHOE with hyphal elements completely penetrating all the epithelial layers (Fig. 1D).

Histological examination of the RHOE after infection with $C$. albicans, demonstrated noticeable changes (Fig. 1D) compared with tissue infected with C. glabrata (Fig. 1A-C). It was evident that $C$. albicans RHOE infection induced superficial detachment of keratinocyte layers (Fig. 1D).

In order to quantify tissue damage, the levels of LDH released by epithelial cells after infection were measured (Fig. 2) and this showed that infection with $C$. albicans produced higher relative levels of LDH activity $(P<0.05)$ compared with $C$. glabrata strains. Furthermore, a moderate but statistically significant increase in relative LDH activity levels was induced by $C$. glabrata strains compared with uninfected controls $(P<0.05)$. Statistical differences with respect to LDH activity released from the RHOE were not evident for C. glabrata RHOE infection $(P>0.05)$.

\section{Candida glabrata and Candida albicans RHOE mixed} species infection

Tissues simultaneously infected with $C$. glabrata and C. albicans were subjected to appropriate histological procedures, and the Candida species differentiated by hybridization with specific $C$. glabrata/C. albicans PNA probes. The relative pattern of infection and ability to invade the RHOE was then observed by CLSM.

Contrary to C. glabrata single species infection (Fig. 1 and Table 1), when co-infected with $C$. albicans, the majority of C. glabrata strains were found to invade the RHOE (Fig. 3 and Table 1). Yeast of oral isolates (C. glabrata D1 and AE2), the urinary isolate (C. glabrata 

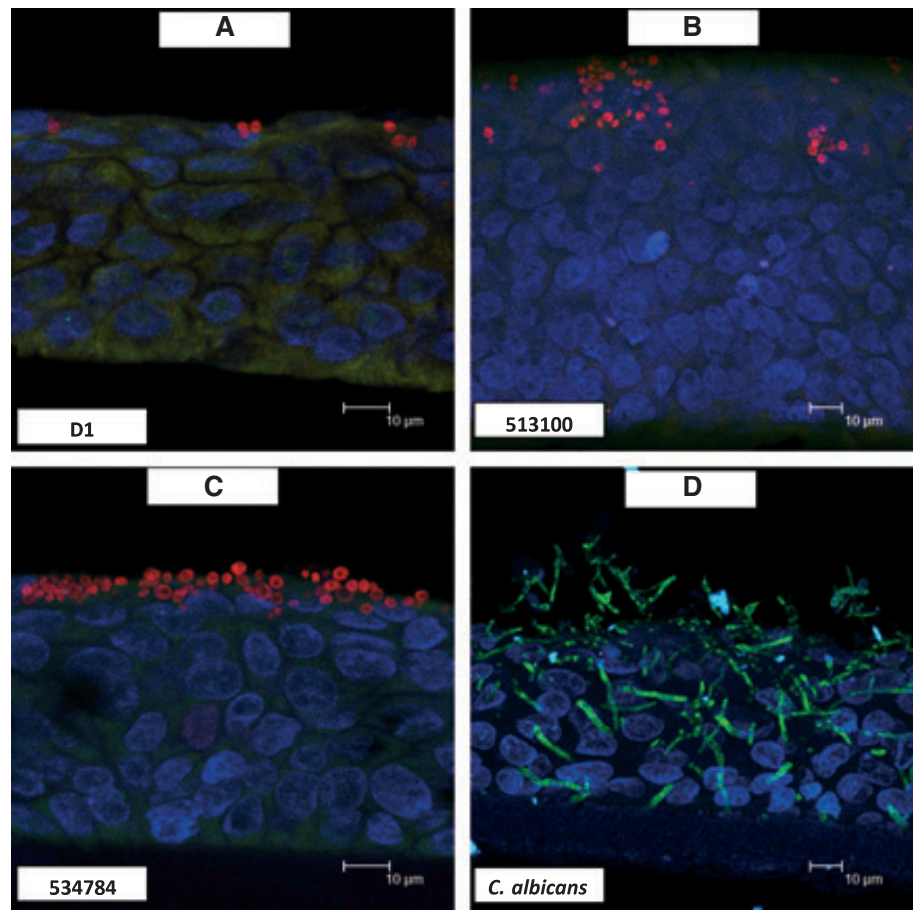

Figure 1 Confocal laser scanner microscopy of Candida strains after $12 \mathrm{~h}$ of RHOE single infection, using YTL PNA FISH ${ }^{\mathrm{TM}}$. C. glabrata cells appear red, C. albicans are green, and keratinocytes nuclei are blue. (A) C. glabrata D1 showing sparse colonization (+) and no invasion; (B) C. glabrata 513100 showing moderate colonization $(++)$ and moderate invasion; $($ C) C. glabrata 534784 showing extensive colonization $(+++)$ and no invasion; (D) C. albicans showing extensive colonization $(+++)$ and high invasion.

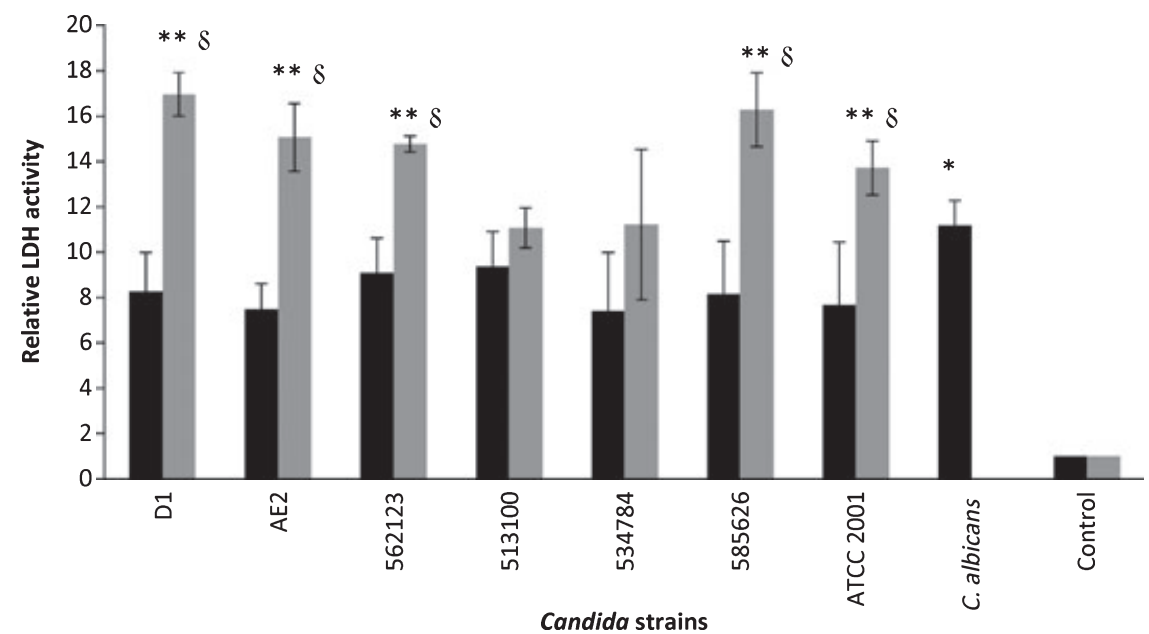

Figure 2 Relative lactate dehydrogenase (LDH) activities measured in the RHOE supernatant after $12 \mathrm{~h}$ of single infection ( $\mathbf{\square})$ and co-infection $(\square)$ with C. glabrata and C. albicans. Control was performed without Candida cells. Error bars represent standard deviation. *LDH activity of Candida albicans single infection is statistically different from $C$. glabrata strains single infections $(P<0.05)$. **LDH activity of co-infection is statistically different from the respective single infection by each $C$. glabrata strain $(P<0.05) .{ }^{\S} \mathrm{LDH}$ activity of $C$. albicans single infection is statistically different from co-infection values $(P<0.05)$.

562123) and the vaginal isolate (C. glabrata 534784) penetrated to the third epithelial layer of the RHOE (Fig. 3A). In the case of C. glabrata 513100 (urinary isolate), C. glabrata 585626 (vaginal isolate) and the reference strain (C. glabrata ATCC 2001) penetration to the lowest epithelium layers (Fig. 3B) was evident. Within the tissue, C. glabrata yeast were localized close to vacuolized tissue portions (Fig. 4A) and observed in close proximity to $C$. albicans hyphal elements (Fig. 4B,C).

For several strains, the LDH levels released by the RHOE epithelial cells infected by both species increased significantly compared to single species infection (Fig. 2). Specifically, for tissues co-infected with C. albicans and the oral isolates (C. glabrata D1 and AE2), the vaginal isolate C. glabrata 585626 and the 
A

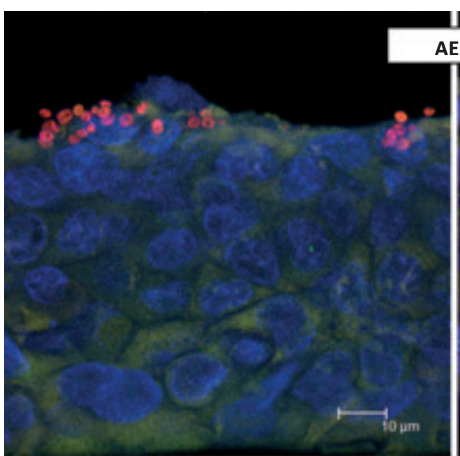

B

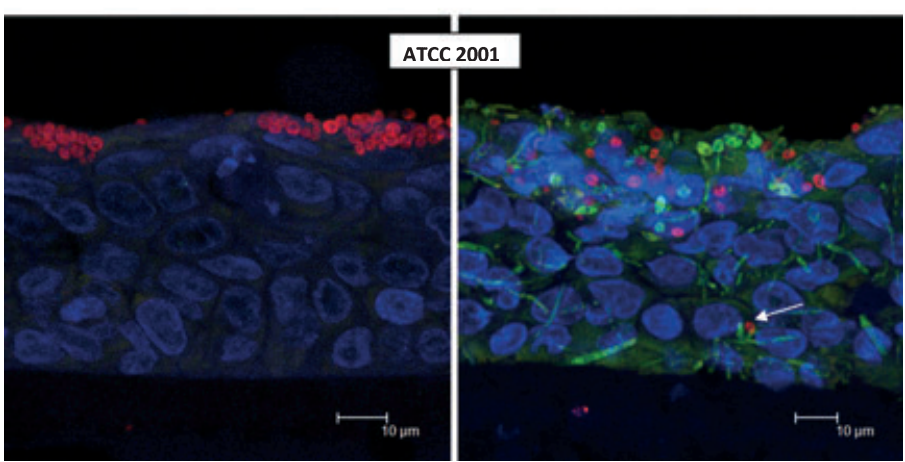

Figure 3 Single (A) and co-infection (B) of C. glabrata and C. albicans of RHOE after $12 \mathrm{~h}$, assessed by CLSM and YTL PNA FISH ${ }^{\mathrm{TM}}$. C. glabrata cells appear red, C. albicans green, and keratinocytes nucleus blue. Candida glabrata AE2 presents moderate invasion while C. glabrata ATCC 2001 presents high invasion in the presence of C. albicans.
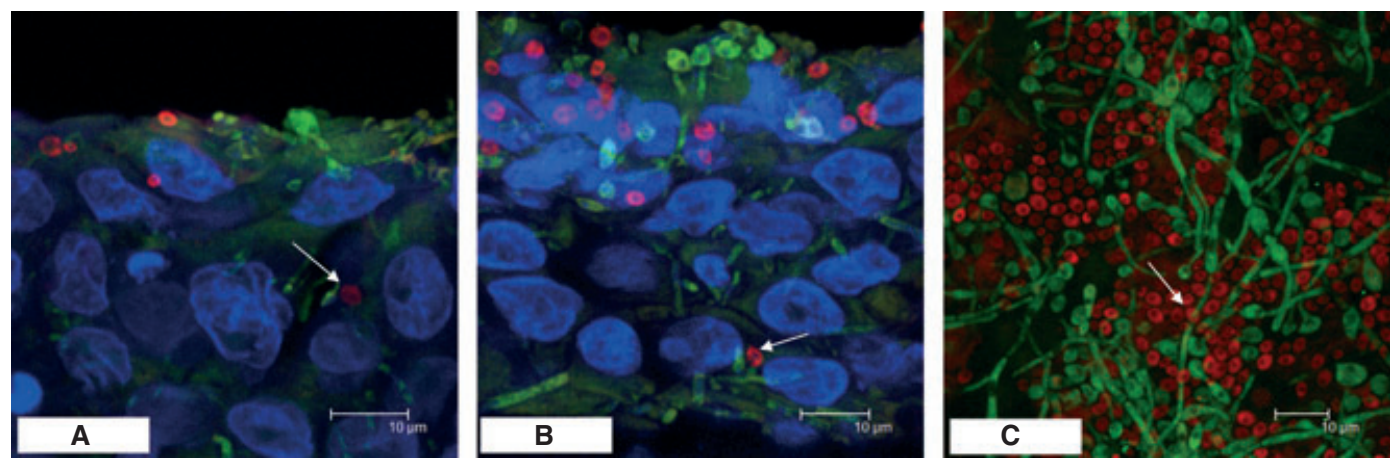

Figure 4 Candida glabrata/Candida albicans co-infection of RHOE after $12 \mathrm{~h}$, assessed by CLSM and YTL PNA FISH ${ }^{\mathrm{TM}}$. Panel A shows the presence of $C$. glabrata yeast at a vacuolized tissue portion (see arrow), panel B illustrates shows C. glabrata cells near $C$. albicans hyphal elements (see arrow) and panel $\mathrm{C}$ shows the adhesion and co-aggregation of $C$. glabrata cells on hyphal forms of $C$. albicans (see arrow).

reference strain (C. glabrata ATCC 2001) the levels of LDH increased approximately two-fold, whilst a slightly lower (1.5-fold) increase was evident with C. glabrata 562123 (urinary isolate). This increase in LDH activity was found to be significant $(P<0.05)$. However, no statistically differences were found in the LDH activity released by single and co-infection of C. glabrata 513100 (urinary isolate) and C. glabrata 534784 (vaginal isolate). Furthermore, an increase in the levels of LDH activity was verified between $C$. albicans single infection and co-infection concerning both oral isolates $(C$. glabrata D1 and AE2), one vaginal isolate (C. glabrata 585626), one urinary isolate (C. glabrata 562123) and the reference strain (C. glabrata ATCC 2001)
$(P<0.05)$. This augment in activity was not found for $C$. albicans co-infecting with one urinary isolate $(C$. glabrata 513100) and one vaginal isolate (C. glabrata 534784) $(P>0.05)$.

\section{Discussion}

Although C. albicans is the most frequently encountered pathogen $(2,3)$, several other species, and especially $C$. glabrata, have recently emerged as oral pathogens (5-7). Candida glabrata is unable to grow in filamentous forms and is therefore only seen as relatively small yeast cells in humans (8). In contrast, C. albicans is polymorphic being able to grow as yeast, pseudohyphae and hyphae 
(25). In terms of tissue invasion, the ability of C. albicans to generate hyphae is seen as advantageous as such filamentous growth enables migration of the fungal element from the point of adherence. Thigmotropic movement is also a property of Candida hyphae (26) which helps to promote invasion of the epithelium (14, $26,27)$. The morphological differences and potentially different patterns of growth exhibited by $C$. albicans and C. glabrata could allow these species to occupy the same location within the oral cavity with limited competition for space, thereby allowing co-existence in many instances.

In recent years, C. albicans pathogenesis in mucosal candidosis has been investigated by several authors (19$21,28)$ using RHOE as a surrogate model to animals. Since mixed infection with both $C$. albicans and $C$. glabrata species has often been reported in the mouth $(5$, 6), we aimed to examine for the first time the effect of mixed infections in the RHOE model. This in turn would indicate the possible pathogenic significance of mixed infection in the oral cavity.

Initially, the colonization patterns and invasion of RHOE by several $C$. glabrata strains originating from different body sites were examined. The results obtained (Table 1, Figs 1A-C and 3A) showed that the surface of the RHOE was successfully colonized by all C. glabrata strains tested, although this was noticeably strain dependent. Furthermore, CLSM revealed that C. glabrata were generally unable to invade the RHOE (Fig. 2). One exception was the urinary isolate $C$. glabrata 513100 which appeared to invade the superficial epithelial layers of the RHOE. These results highlight the diversity of $C$. glabrata strains in colonization and invasiveness capability, and mirror those previously reported for C. parapsilosis (22) and C. albicans (21). Schaller et al. (27) and Jayatilake et al. (20) have both previously classified C. glabrata strains as being noninvasive in RHOE infection.

It is important to note that six of the seven strains analysed in this present study and classified as 'noninvaders' were sourced from different body sites, possibly indicating that strain origin is not an important aspect in C. glabrata colonization and invasion of oral epithelium. For example, the oral isolates (AE2 and D1) exhibited a lower ability to colonize the RHOE, compared with vaginal isolates (534784 and 585626).

Generally, most $C$. albicans isolates infecting the RHOE model exhibit histological signs of hyphal invasion $(14,19,25,27)$. This current study also confirmed the high invasiveness of $C$. albicans (Fig. 1D) and particularly so when compared with $C$. glabrata (Figs $1 \mathrm{~A}-\mathrm{C}$ and $3 \mathrm{~A}$ ). In fact, after $12 \mathrm{~h}$ of infection, invasion of the RHOE by hyphal elements of C.albicans was clearly evident, leading to marked disorganization of RHOE and detachment of superficial layers of the tissue from the insert (Fig. 1D); this was in contrast to the lower level of damage caused by $C$. glabrata strains (Figs 1A-C and 3A). The extent of tissue damage was quantitatively assessed by measurement of relative $\mathrm{LDH}$ activity (Fig. 2). The results were in accordance with the extent of Candida colonization and tissue damage observed histologically (Fig. 1). The levels of LDH released during single species infection was highest for $C$. albicans which was expected given the previous histological observations and considering that this species is generally the most virulent $(19,27)$.

Interestingly, mixed species infection revealed (Table 1 and Fig. 3B) that C. albicans promoted the invasiveness of C. glabrata. In fact, all six C. glabrata strains classified as 'non-invaders' in single infection studies were found to penetrate the oral epithelium in the presence of $C$. albicans. It is known that $C$. albicans hyphal formation is a critical determinant in invasive pathogenesis especially in comparison with non-Candida albicans Candida species, and it has been shown that $C$. albicans with mutations in genes involved in promoting hyphal development were less invasive than wild type strains (20).

The exact mechanism of how $C$. albicans might promote C. glabrata penetration of the oral epithelium remains unclear. Damage to the integrity of the epithelial surface caused by the growing tips of the $C$. albicans hyphae (Fig. 4A,B) seems to provide access to lower epithelial layers for C. glabrata yeast. In fact, C. glabrata cells were observed in close proximity to $C$. albicans hyphae and transported in this adhered form to deeper tissue layers (Fig 4C, arrows). In this context, Ez-Azizi et al. (29) showed the efficient adherence of $C$. albicans to a pre-formed C. glabrata biofilm in a catheter model, suggesting the co-aggregation of these two species in vivo.

The increased LDH activity (Fig. 2) reported after mixed infection with $C$. albicans and $C$. glabrata supports a finding of enhanced pathogenicity (7). Of additional concern is that strains of $C$. glabrata often have higher resistance to azoles antifungal agents compared with many other Candida species, including C. albicans $(5,6,30)$. In such instances, mixed infection would not only exhibit enhanced pathogenicity (7), but could also be more problematic to treat. Many diagnostic laboratories are limited in the identification of mixed Candida infection, particularly when non-differential primary isolation agars such as Sabouraud dextrose agar are used. The importance of recognizing the occurrence of such mixed infection is however highlighted by the results of this study and represents an area that should be considered by both diagnostic laboratories and clinicians alike.

\section{References}

1. Samaranayake LP. Host factor and oral candidosis. In: Samaranayake LP, Macfarlane TW, eds. Oral candidosis. London, UK: Butterworth \& Co. (Publishers) Ltd, 1990; 66-103.

2. Pfaller MA, Diekema DJ, Jones RN, Messer SA, Hollis RJ. Trends in antifungal susceptibility of Candida spp. isolated from pediatric and adult patients with bloodstream infections: SENTRY Antimicrobial Surveillance Program, 1997 to 2000. J Clin Microbiol 2000; 40: 852-6.

3. Trick WE, Fridkin SK, Edwards JR, Hajjeh RA, Gaynes RP. National Nosocomial Infections Surveillance System 
Hospitals. Secular trend of hospital-acquired candidemia among intensive care unit patients in the United States during 1989-1999. Clin Infect Dis 2002; 35: 627-30.

4. Li L, Redding S, Dongari-Bagtzoglou AD. Candida glabrata, an emerging oral opportunistic pathogen. J Dent Res 2007; 86: 204-15.

5. Redding SW, Kirkpatrick WR, Dib O, Fothergill AW, Rinaldi MG, Patterson T. The epidemiology of nonalbicans Candida in oropharyngeal candidiasis in HIV patients. Spec Care Dentist 2000; 20: 178-81.

6. Redding SW, Kirkpatrick WR, Coco BJ, et al. Candida glabrata oropharyngeal candidiasis in patients receiving radiation treatment for head and neck cancer. $J$ Clin Microbiol 2002; 40: 1879-81.

7. Coco BJ, Bagg J, Cross LJ, Jose A, Cross J, Ramage G. Mixed Candida albicans and Candida glabrata populations associated with pathogenesis of denture stomatitis. Oral Microbiol Immunol 2008; 23: 377-83.

8. Fidel PL, Vazquez JA, Sobel JD. Candida glabrata: review of epidemiology, pathogenesis, and clinical disease with comparison to C. albicans. Clin Microbiol Rev 1999; 12: 8096.

9. Canuto MM, Gutierrez RF, Ortiz TDV, et al. Determinants for the development of oropharyngeal colonization or infection by fluconazole-resistant Candida strains in HIV-infected patients. Eur J Clin Microbiol Infect Dis 2000; 19: 593-601.

10. Olmos MA, Araya V, Concetti H, et al. Oesophageal candidiasis: clinical and mycological analysis. Acta Gastroenterol Latinoam 2005; 35: 211-8.

11. Miranda LN, van der Heijden IM, Costa S, et al. Candida colonisation as a source for candidaemia. J Hosp Infect 2009; 72: 9-16.

12. Hayden RT, Qian X, Roberts GD, Lloyd RV. In situ hybridization for the identification of yeast like organisms in tissue section. Diagn Mol Pathol 2001; 10: 15-23.

13. Hayden RT, Qian X, Procop GW, Roberts GD, Lloyd RV. In situ hybridization for the identification of filamentous fungi in tissue section. Diagn Mol Pathol 2002; 11: 119-26.

14. Malic S, Hill KE, Hayes A, et al. Detection and identification of specific bacteria in wound biofilms using peptide nucleic acid (PNA) fluorescent in situ hybridization (FISH). Microbiology 2009; 155: 2603-11.

15. Oliveira K, Procop GW, Wilson D, Coull J, Stender H. Rapid identification of Staphylococcus aureus directly from blood cultures by fluorescent in situ hybridization with peptide nucleic acid probes. J Clin Microbiol 2002; 40: 247-51.

16. Guimarães N, Azevedo NF, Figueiredo C, Keevil CW, Vieira MJ. Development and application of a novel peptide nucleic acid probe for the specific detection of Helicobacter pylori in gastric biopsy. J Clin Microbiol 2007; 45: 3089-94.

17. Reller ME, Mallonee AB, Kwiatkowski NP, Merz WG. Use of PNA-FISH for definitive, rapid identification of five common species of Candida. J Clin Microbiol 2007; 45: $3802-3$.
18. Shepard JR, Addison RM, Alexander BD, et al. Multicenter evaluation of the $C$. albicans/C. glabrata PNA FISH method for simultaneous dual color identification of Candida albicans and Candida glabrata directly from blood culture bottles. J Clin Microbiol 2008; 46: 50-5.

19. Bartie KL, Williams DW, Wilson MJ, Potts AJ, Lewis MA. Differential invasion of Candida albicans isolates in an in vitro of oral candidosis. Oral Microbiol Immunol 2004; 19: 293-6.

20. Jayatilake JA, Samaranayake YH, Cheung LK, Samaranayake LP. Quantitative evaluation of tissue invasion by wild type, hyphal and SAP mutants of Candida albicans, and non-albicans Candida species in reconstituted human oral epithelium. J Oral Pathol Med 2006; 35: 484-91.

21. Malic S, Hill KE, Ralphs JR, et al. Characterization of Candida albicans infection of an in vitro oral epithelial model using confocal laser scanning microscopy. Oral Microbiol Immun 2007; 22: 188-94.

22. Silva S, Henriques M, Oliveira R, et al. Characterisation of Candida parapsilosis infection of an in vitro reconstituted human oral epithelium. Eur J Oral Sci 2009; 117: 669-75.

23. Silva S, Hopper SJ, Henriques M, Oliveira R, Azeredo J, Williams DW. The role of secreted aspartyl proteinases in Candida tropicalis invasion and damage of oral mucosa. Clin Microbiol Infect 2010; Doi:10.1111/j.1469-0691.2010. 03248

24. Williams DW, Wilson MJ, Lewis MA, Potts AJ. Identification of Candida species by PCR and restriction fragment length polymorphism analysis of intergenic spacer regions of ribosomal DNA. J Clin Microbiol 1995; 33: 2476-9.

25. Odds FC. Candida and Candidosis, 2nd edn. London: Bailliere Tindall, 1998.

26. Gow N. Fungal morphogenesis: some like it hot. Curr Biol 2009; 19: 621-9.

27. Schaller M, Mailhammer R, Grassl G, Sander CA, Hube B, Korting HC. Infection of human oral epithelia with Candida species induces cytokine expression correlated to the degree of virulence. $J$ Invest Dermatol 2002; 118: 652-7.

28. Schaller M, Zakikhany K, Naglik Jr, Weindl G, Hube B. Models of oral and vaginal candidiasis based on in vitro reconstituted human epithelia. Nat Protoc 2006; 1: 2767-73.

29. El-Azizi MA, Starks SE, Khardori NJ. Interactions of Candida albicans with other Candida spp, and bacteria in the biofilms. Appl Microbiol 2004; 96: 1067-73.

30. Bagg J, Sweeney MP, Lewis MA, Jackson MS, Coleman D, Mosaid A. High prevalence of non-albicans yeasts and detection of antifungal resistance in the flora of patients with advanced cancer. Palliat Med 2003; 17: 477-81.

\section{Acknowledgements}

We would like to thank Mrs Kath Allsopp for processing and sectioning tissue samples. The authors also would like to thank Mr Mark Fiandaca of AdvanDX for provision of Yeast Traffic Light PNA FISH ${ }^{\mathrm{TM}}$ Kit. This work was supported by the research grant SFRH/BD/28341/2006 from the Fundação para a Ciência e Tecnologia (FCT), Portugal. 